\title{
1 Pleiotropy drives repeatability in the genetic basis of adaptation
}

2 Paul Battlay ${ }^{1}$, Sam Yeaman ${ }^{2}$, Kathryn A. Hodgins ${ }^{1}$

3 1. School of Biological Sciences, Monash University, Melbourne, Victoria, Australia

4 2. Department of Biological Sciences, University of Calgary, Calgary, Alberta, Canada

\section{Abstract}

7 Studies of trait-mapping and local adaptation often identify signatures of genetically parallel 8 evolution, where different species evolve similar phenotypes using the same genes. Such 9 patterns appear incongruent with current estimations of quantitative trait architecture. With 10 hundreds or thousands or genes contributing to a trait, why would selection make repeated use

11 of the same genes? Here, we use individual-based simulations to explore a two-patch model

12 with quantitative, pleiotropic traits to understand the parameters which may lead to repeated use

13 of a particular locus during independent bouts of adaptation. We find that repeatability can be

14 driven by increased phenotypic effect size, a reduction in trait dimensionality and a reduction in

15 mutational correlations at a particular locus relative to other loci in the genome, and that these

16 patterns are magnified by increased migration between demes. These results suggest that

17 evolutionary convergence can arise from multiple characteristics of a locus, and provide a

18 framework for the interpretation of quantitative signatures of convergence in empirical studies.

20 Keywords: pleiotropy, parallel evolution, repeatability, migration, simulations 


\section{Introduction}

22 Studies of adaptation commonly observe convergent genetic responses, where multiple species

23 independently respond to a given selection pressure with mutations in orthologous genes.

24 These patterns imply a lack of redundancy in the genes available for a selective response, and

25 at first glance seem inconsistent with another common observation: that variation in quantitative

26 traits is explained by a very large number of alleles of small effect, which suggests a high level

27 of redundancy in the genes contributing to quantitative traits.

In the early 20th century, theoretical work by R. A. Fisher demonstrated that the continuous phenotypic variation observed in populations could be explained by a large number of alleles inherited in a Mendelian manner (Fisher 1918), and that selection would favor small-effect changes at large numbers of loci (Fisher 1930). Genome-wide association studies in humans have provided empirical observations of standing variation congruent to Fisher's models of adaptive trait architecture (reviewed in Visscher et al. 2017): Associations with hundreds or thousands of genetic variants explain only a modest proportion of trait heritability, with the remaining heritability attributable to even larger numbers of variants with effect sizes too small to detect with current cohorts (or possibly to rare variants that are excluded from many such analyses). But if variation in thousands of genes underpins a given trait, why would we ever observe orthologous genes contributing to adaptation in multiple species, when there are seemingly a myriad of ways to construct the same traits?

In his revisiting of Fisher's model, Kimura (1968) demonstrated that although smaller effect mutations are more likely to be favourable, beneficial mutations of small effect are less likely to fix, as genetic drift biases the contribution of intermediate-effect loci to adaptation. Later, Orr (1998) showed that effect sizes of fixed adaptive mutations during an adaptive walk should be exponential, illustrating the importance of large-effect mutations early in bouts of adaptation to sudden environmental change. The omnigenic model (which posits that all genetic variants in genes expressed in the relevant cell type contribute to a phenotype; Boyle, Li \& Pritchard 2017; Liu, Li \& Pritchard 2019) also makes the distinction between 'core' genes of larger effect and 'peripheral' genes of small effect (although the latter explains the bulk of trait heritability). Perhaps the simplest explanation for convergent genetic adaptation is if alleles of large effect

52 are disproportionately likely to contribute to adaptation (e.g., because of their fixation

53 probabilities), but only a subset of loci are able to generate alleles of large effect (Orr 2005).

54 Convergence in gene use would then occur if there is long-term conservation of the genotype- 
55 phenotype map and the potential for particular loci to generate alleles of large effect. Certainly,

56 large-effect QTL have been identified in both experimental evolution studies (e.g. McKenzie \&

57 Batterham 1994) and natural populations (e.g. Shapiro et al. 2004; Doebly et al. 2004), and

58 genomic footprints of selective sweeps (Smith \& Haigh 1974; Kaplan, Hudson \& Langley 1989)

59 provide evidence for strong selection at individual loci. The effects of local adaptation on genetic

60 architecture may further act to increase the likelihood of repeatability, as the contributions of

61 small-effect alleles are disproportionately limited by the swamping effect of gene flow in

62 populations connected by migration (Yeaman \& Whitlock 2011). Consequently, convergence in

63 the genetic basis of local adaptation is expected to frequently involve large-effect mutations,

64 particularly when gene flow is high or drift is strong, yet these processes do not overwhelm

65 selection (Yeaman et al. 2018).

67 While alleles of large effect may be favoured early in adaptation or when there is migration-

68 selection balance, their contribution to adaptation can be limited by pleiotropy.

69 In both Fisher (1930) and Orr's models (1998), mutations are modelled as vectors in

70 multidimensional phenotypic space; therefore mutations with a large effect in a favorable

71 dimension generally deviate too far from the optima in other dimensions to increase overall

72 fitness. Chevin, Martin \& Lenormand (2010) expanded these models to incorporate distinct

73 genes which could vary in their pleiotropic properties: specifically the number of traits that

74 mutations would affect, and the correlation in effects of mutations on different traits (the latter

75 being a property that can arise from organization of genes into networks; Hether \& Hohenlohe

76 2014). They demonstrated that repeatability in the genetics of adaptation is an expected

77 consequence of between-locus variation in pleiotropy; convergence may therefore be observed

78 in genes where negative pleiotropic effects are minimized.

Neither local adaptation nor pleiotropy has been extensively studied in terms of their effects on

81 repeatability, so if we are to interpret empirical observations of repeatability, we need a solid

82 grounding in this theory. Here, we utilize individual-based simulations of quantitative trait

83 evolution to understand how the interplay between inter-locus heterogeneity in pleiotropy and

84 migration-selection balance affects genetic convergence. We build on previous models, which

85 have considered adaptation in a single population following an environmental shift, by

86 introducing a second population adapting to a divergent environment, allowing the observation

87 of interactions between migration, effect size and pleiotropy in bouts of local adaptation. We

88 find that increasing effect size or decreasing pleiotropy (both the overall dimensionality as well 
bioRxiv preprint doi: https://doi.org/10.1101/2021.09.13.459985; this version posted September 13, 2021. The copyright holder for this preprint (which was not certified by peer review) is the author/funder, who has granted bioRxiv a license to display the preprint in perpetuity. It is made available under aCC-BY-NC-ND 4.0 International license.

89 as mutational correlation) at a given QTL relative to the other QTL will increase repeatability.

90 Moreover we find that increased migration between demes exacerbates the repeatability

91 observed. 


\section{Simulations}

93 To study the factors driving repeatability at particular loci in independent bouts of adaptation, we

94 used SLiM software (Haller \& Messer 2019) to simulate adaptation to a complex environment

95 that varied across two patches connected by migration. Adaptation within each patch was

96 driven by selection on two (or more) traits: $Z_{i}$ with an optimum that varied among the patches,

97 and one or more (e.g. $Z_{j}$ ) with the same optimum in each patch.

Traits could be affected by mutations at five genetically unlinked QTL, four QTL with uniform properties and a single QTL with aberrant properties: At one 'focal' QTL, parameter values could be varied independently of the 'non-focal' QTL. For some parameters, simulations were repeated with a total of 20 QTL and one focal QTL (fig. S3). Each QTL consisted of 500bp, and mutations occurred at a rate of $1 \times 10^{-7}$, resulting in an expected 10,000 mutations in each of two demes over the 20,000 -generation simulation.

QTL mutations affected two or more phenotypes (e.g. $Z_{i}$ and $Z_{j}$ ); mutational effects for each QTL were drawn from a multivariate normal distribution where the variance was equal to the QTL effect magnitude and the covariance was equal to the QTL mutational correlation multiplied by the effect magnitude.

The following Gaussian function related individual fitness to phenotype:

$$
e^{-\frac{(\theta-\Sigma a)^{2}}{2 V_{S}}}
$$

114 where $\theta=$ the phenotypic optimum, $\Sigma a=$ the sum of mutation effects, and $V_{s}=$ the variance in 115 the fitness function, reflecting the strength of stabilizing selection (set at 125 for all simulations). 116 Overall individual fitness was calculated as the product of fitness values across all phenotypes, 117 and there was no correlational selection between pairs of phenotypes. We simulated two demes ( $d_{1}$ and $d_{2}$ ), each composed of 1000 randomly-mating hermaphroditic

120 individuals. Phenotypic space was unitless and provided a relative scaling of fitness vs 121 mutational effect. Both demes began the simulation with phenotypic optima of 0 for all 122 phenotypes and ran with a burn-in for 20,000 generations. After the burn-in, in $d_{1}$ the optima for 
123 all phenotypes remained at 0 , while in $d_{2}$, the optimum for $Z_{i}$ was shifted to -10 , while other

124 phenotypic optima remained at 0 , and we tracked adaptive evolution over the following 20,000

125 generations. We varied the migration rate between $d_{1}$ and $d_{2}$ (from 0 to 0.05 ) and the mutation

126 effect magnitudes (from 0.1 to 5), mutational correlations (from 0 to 0.99 ), and the number of

127 phenotypes affected at the QTL.

128

129 To interpret the results of each parameter combination, we calculated the genetic value (GV) of 130 each mutation to a phenotype using the formula:

$$
G V=\left(p_{1}-p_{2}\right) \times a
$$

133 where $p_{1}$ and $p_{2}$ are the frequencies of a mutation in each deme, and $a$ is the size of the

134 mutation's effect on $Z_{i}$

136 We investigated three main ways in which the characteristics of the focal QTL could be 137 differentiated from those of the other loci:

1) A change in effect magnitude by altering the variance component of the variancecovariance matrix used to generate mutations (fig. 1A cf. B). This parameter was used to model a large-effect QTL at the focal QTL.

2) A change in mutational correlation by altering the covariance component of the variance-covariance matrix (fig $1 \mathrm{~A}$ cf. $\mathrm{C}$ ). This parameter models dependence between phenotypes and determines the likelihood that a mutation's effect on one phenotype will have a corresponding effect on another.

3) A change in the number of phenotypes affected by a mutation by reducing the dimensionality of the variance-covariance matrix (fig. 1A cf. D). This models a situation where a QTL has no effect on one or more phenotypes.. 

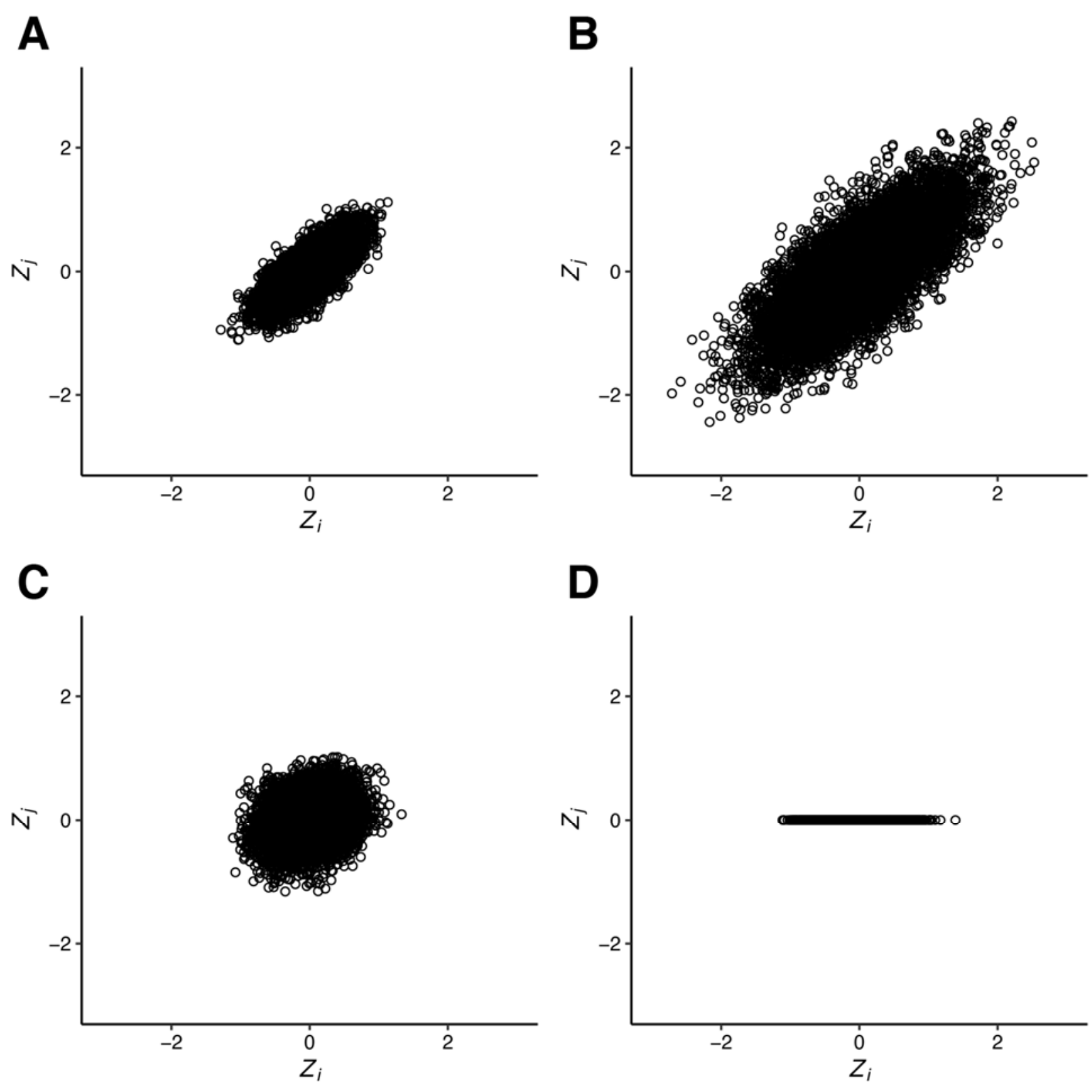

Figure 1. Effect sizes on $Z_{i}$ (x-axes) and $Z_{j}$ (y-axes) for 10,000 draws from distributions used to generate mutations. In $A$, the mean effect magnitude is 0.1 and the mutational correlation between traits is 0.75 .

151 In $B$, the mutational correlation is the same as $A(0.75)$ but the mean effect magnitude is increased to 0.5 . 152 In $\mathrm{C}$, the mean effect magnitude is the same as $\mathrm{A}(0.1)$, but the mutational correlation is relaxed to 0.25 . 153 In $\mathrm{D}$, mutations have no effect on the non-divergent phenotype. 
154 For each parameter combination we quantified the divergence between demes $d_{1}$ and $d_{2}$ at the

155 divergently selected phenotype by summing $2 \times G V$ across all individuals, and quantified

156 repeatability in the contributions of the QTL to trait divergence (measured by QTL-specific GV)

157 across 100 replicates using the $C_{c h i s q}$ statistic with 1000 permutations (Yeaman et al. 2018).

158 Briefly, $\mathrm{x}^{2}$ was calculated across simulation replicates with:

159

$$
\chi^{2}=\frac{\Sigma\left(\underline{\alpha_{i}}-\underline{\alpha}\right)^{2}}{\underline{\alpha}}
$$

161 where $\underline{\alpha_{i}}$ is the sum across simulation replicates of $G V$ for the $i^{\text {th }} \mathrm{QTL}$, and $\underline{\underline{\alpha}}$ is the mean $\underline{\alpha}$ 162 across all QTL.

163

164 The $C_{\text {chisq }}$ statistic was then calculated by using $\chi^{2}$ and $\chi_{\text {sim }}^{2}$, the results of 1000 permutations of 165 the data within each replicate:

166

$$
C_{\text {chisq }}=\frac{\chi^{2}-\operatorname{mean}\left(\chi_{\text {sim }}^{2}\right)}{s d\left(\chi_{\text {sim }}^{2}\right)}
$$

168 By this equation, when $C_{\text {chisq }}=0$ we observe no excess repeatability, and complete repeatability 169 is observed for five QTL when $C_{c h i s q}=2$.

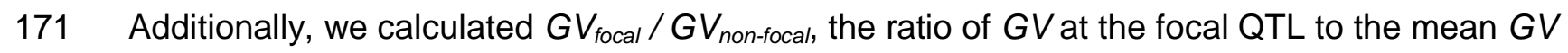
172 across non-focal QTL. 


\section{Results}

174 To model the case where the focal QTL is more important to the divergent phenotype than the 175 non-focal QTL, we examined the effect of varying the effect magnitude at the focal QTL while 176 holding effect magnitude constant at non-focal QTLs. The genes involved in adaptation are

177 random $\left(C_{c h i s q}=0\right)$ when all loci have the same mutation effect size and correlation (fig. 2 where

178 focal QTL effect magnitude $=0.1$ ). Increased repeatability was observed with any increase in 179 focal QTL effect magnitude (fig. 2) across all mutational correlation and migration rate values.

180 Additionally, increasing migration rates resulted in increasing repeatability (fig. 2; fig. S1), and 181 this pattern was exacerbated by increasing mutational correlations. 


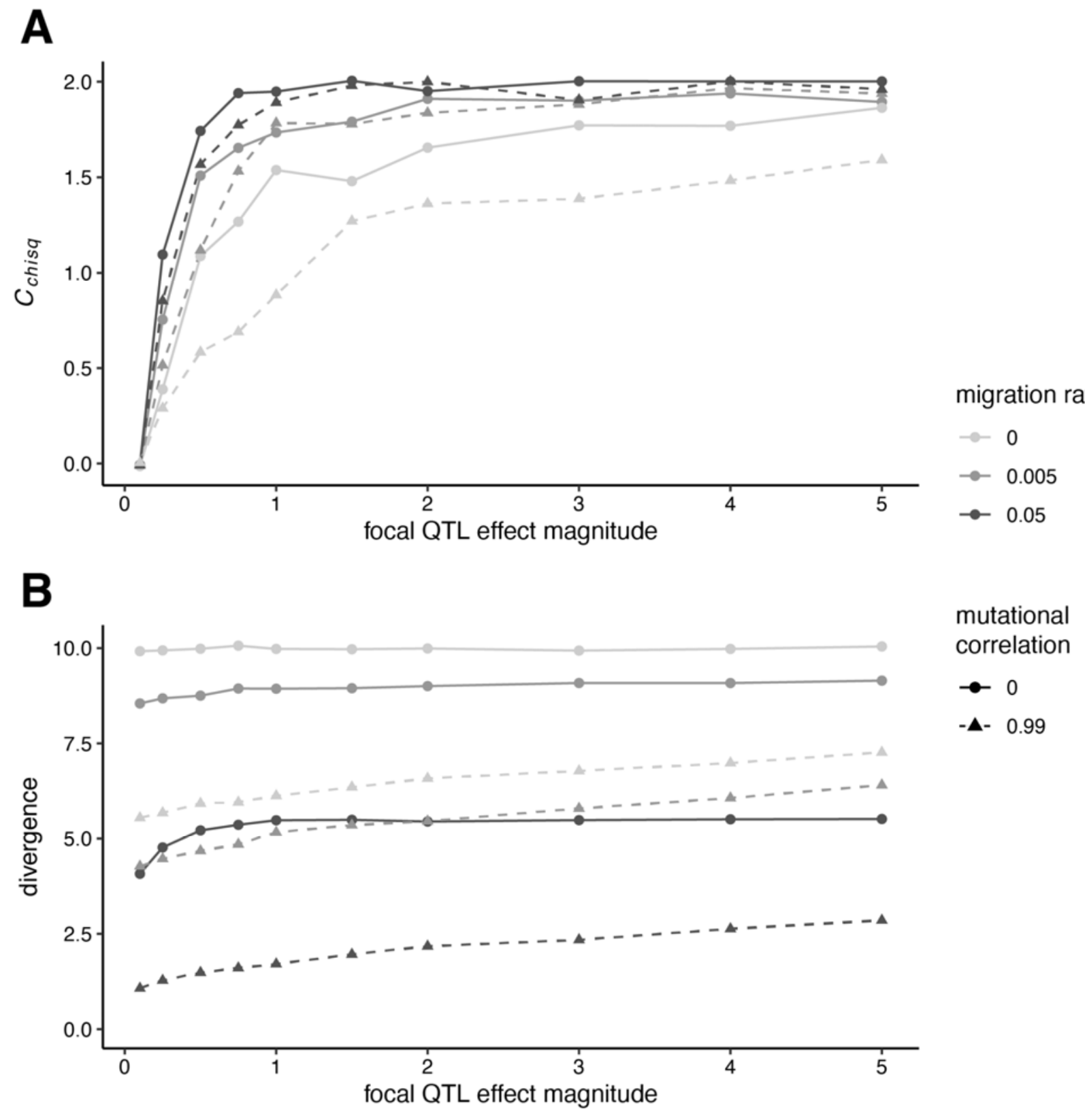

Figure 2. Repeatability $\left(C_{c h i s q}\right)$ in $Z_{i}(\mathrm{~A})$ and $Z_{i}$ phenotypic divergence between $d_{i}$ and $d_{j}(\mathrm{~B})$ against focal 184 QTL effect magnitude where the effect magnitude for non-focal QTL is 0.1 , and where mutational correlations between phenotypes at all QTL are 0 (circle points; solid lines) or 0.99 (triangle points;

186 dashed lines). These simulations use two phenotypes (one divergent and one non-divergent), and were 187 run for 20,000 generations. 
188 Reducing the correlation in phenotypic effects at a QTL may also allow it to more readily acquire

189 adaptive mutations when the direction of change toward the optimum is not aligned with the

190 correlation in phenotypic effects. We modeled this by independently varying mutational

191 correlations at the focal and non-focal QTL (fig. 3; fig. S2), and observed repeatability for

192 mismatches between mutational correlation values (fig. 3). When the mutational correlation at

193 the focal QTL was reduced relative to the non-focal QTL, repeatability involving the focal QTL

194 increased, and when the mutational correlation at the focal QTL was increased relative to the

195 non-focal QTL, repeatability involving the focal QTL decreased (this latter observation was not

196 robust to an increase in the number of QTL [fig. S3]). High levels of repeatability were only

197 seen when the focal QTL had a relaxed mutational correlation against a background of high

198 mutational correlation at non-focal QTL (i.e. 0.75 and particularly 0.9 ). This reflects the fact that

199 mutational correlations need to be high to significantly limit the availability of fortuitous

200 combinations of effects on the phenotypes at the other QTL. 


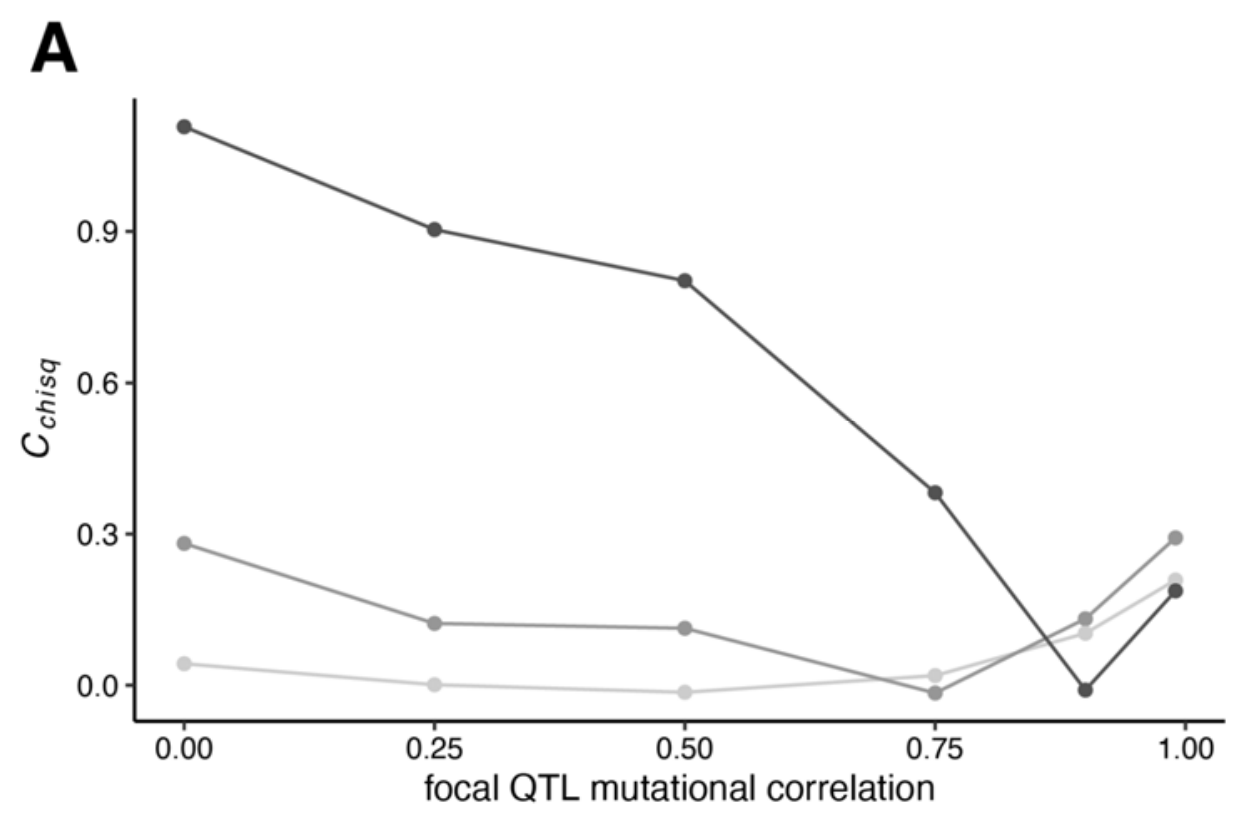

B

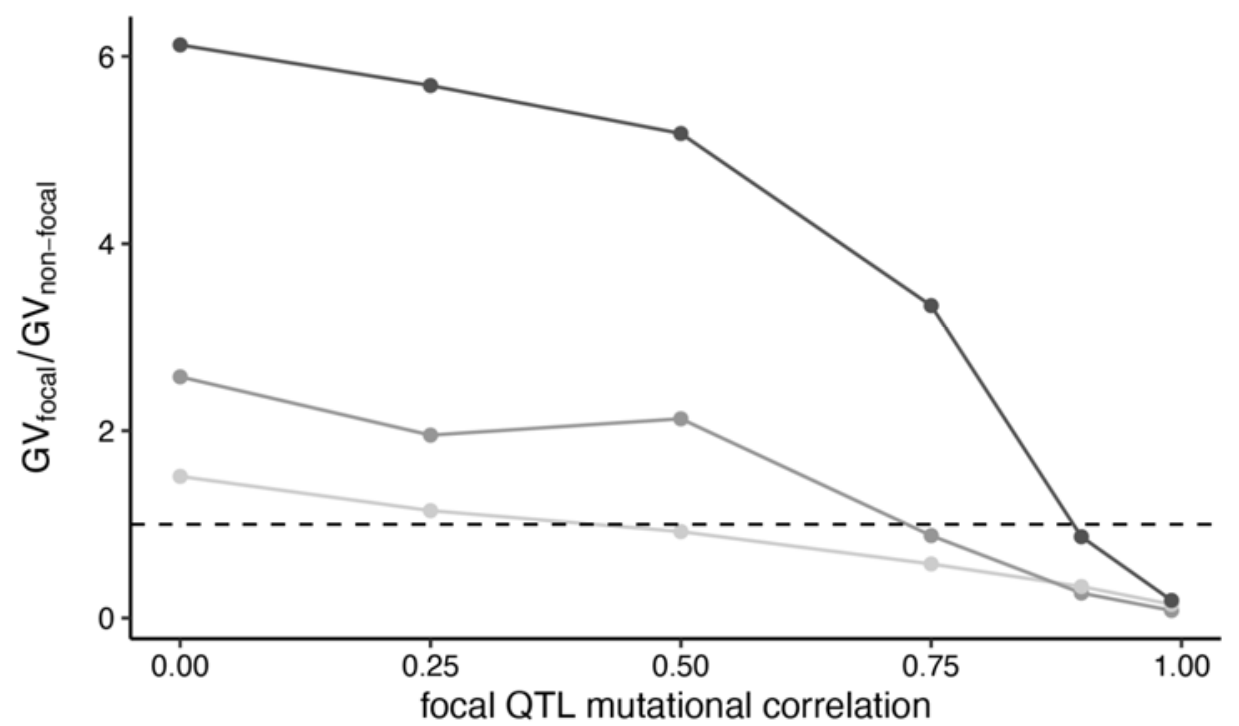

non-focal QTL mutational correlation

$-0.5$

$\rightarrow 0.75$

202 Figure 3. Repeatability $\left(C_{\text {chisq }}\right)$ in $Z_{i}$ against focal QTL mutational correlation for varying values of non203 focal QTL mutation correlation (A), and corresponding ratios of the $G V$ at focal QTL to mean GV at nonfocal QTL (B). The dotted line indicates $G V_{\text {focal }} / G V_{\text {non-focal }}=1$, the point at which this value shifts from representing overuse of the focal QTL to underuse of the focal QTL. These simulations use a migration rate of 0.005 , a mutation effect magnitude of 0.5 and two phenotypes (one divergent and one nondivergent), and were run for 20,000 generations. 


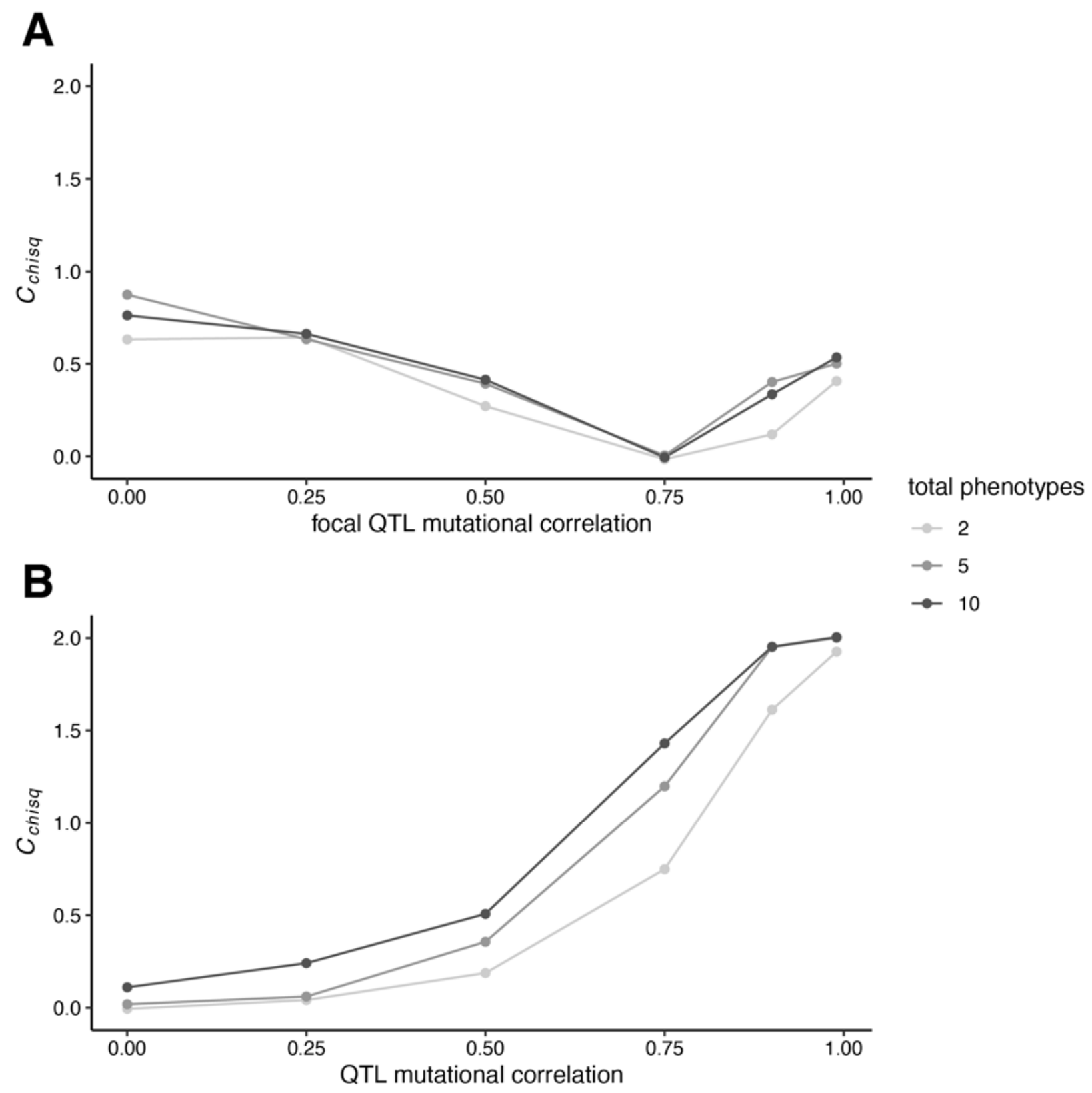

Figure 4. Repeatability ( $\left.C_{\text {chisq }}\right)$ against focal QTL mutational correlation where the non-focal QTL

210 mutation correlation $=0.75(A)$ and repeatability against $Q T L$ mutational correlation where the focal QTL

211 affects the divergent phenotype $\left(Z_{i}\right)$ and one fewer non-divergent phenotypes than the non-focal QTL (B).

212 Shades indicate the total number of phenotypes in the simulation (two with one non-divergent phenotype,

213 five with four non-divergent phenotypes and ten with nine non-divergent phenotypes). These simulations

214 use a migration rate of 0.005 and a mutation effect magnitude of 0.1 , and were run for 20,000

215 generations. 
216 To assess how robust these observations were to an increase in the dimensionality of the

217 model, we increased the number of non-divergent phenotypes from one to nine for the case

218 where the non-focal QTL mutational correlation $=0.75$, but saw only a very modest increase in

219 repeatability (fig. 4A). Finally, we investigated the case where mutations at the focal QTL affect

220 fewer phenotypes than the non-focal QTL. In the two-phenotype model, this meant focal QTL

221 mutations would only affect the divergent phenotype; in the five and ten-phenotype models,

222 focal QTL mutations affected the divergent phenotype and one fewer non-divergent phenotypes

223 than non-focal QTL. With high mutational correlation between phenotypic effects, high levels of

224 repeatability at the focal QTL is observed, however when mutational correlations are weak or

225 absent, very little repeatability is observed (fig. 4B). 


\section{Discussion}

227 Empirical observations of convergent genetic evolution are common (reviewed in Conte et al.

228 2012), but in many ways at odds with some models of complex trait architecture. In this study

229 we used simulations to understand the factors that could be varied at a QTL to produce

230 convergent evolutionary patterns. Firstly, we demonstrated that an increase in effect magnitude

231 of a QTL will produce patterns of repeatability, which is consistent with previous theoretical

232 (Chevin, Martin \& Lenormand 2010) and empirical observations (e.g. Rosenblum, Hoekstra, \&

233 Nachman 2004; Schlenke \& Begun 2004). Both mutational correlations and migration can force

234 adaptation away from phenotypic optima along 'genetic lines of least resistance' (Schluter 1996;

235 Guillaume 2011). Correspondingly, we see a reduction in divergence between demes as

236 mutational correlations or migration is increased (fig. 2B). However, while increasing mutational

237 correlations reduce repeatability, migration amplifies it (fig. 2A).

We also investigated how varying pleiotropy at the focal locus affected signatures of repeatability. Pleiotropy was varied in two ways: a relaxation in mutational correlations with a non-divergent phenotype, or a reduction in the number of phenotypes that a QTL mutation affects. Congruent with the findings of Chevin, Martin \& Lenormand (2010), we found that variation in different forms of pleiotropy will increase the likelihood that repeatability will emerge. Specifically, we find that a reduction in pleiotropic dimensionality at a focal QTL produces greater levels of repeatability than a relaxation in mutational correlations, a pattern that is robust to increases in trait dimensionality in our models (fig. 4A c.f. B). divergent selection, our simulations used two demes linked by varying amounts of migration.

250 This models a common situation in local adaptation: Individuals in one population may experience local environmental shifts; they must therefore adapt to new optima for some phenotypes, while retaining existing optima at others. Previously, Yeaman \& Whitlock (2011) demonstrated that migration concentrates the genetic architecture of local adaptation and favors alleles of larger effect. Correspondingly, we find that migration increases the observed repeatability arising from effect-magnitude variation (fig. 2, fig. S1), as high migration rates favour adaptation by larger effect alleles, which can most readily occur at the focal locus when pleiotropy is present. But this effect breaks down at high migration, where swamping tends to

258 prevent persistent divergence. We also find that migration increases repeatability arising from 259 pleiotropic variation (fig. S2). This is because the net effect of selection on a QTL is driving 
repeatability. Under migration-selection balance those QTL with larger net beneficial effects (weaker mutational correlations) will be maintained as differentiated when there is migration (unless migration is so high that no mutations meet the threshold).

Guillaume (2011) utilized a similar two-patch design to investigate the effects of pleiotropy and migration on population divergence. He demonstrated that combinations of migration and pleiotropy can drive divergence between demes at phenotypes that share the same optima in both demes, as long as the phenotypes are sufficiently correlated with divergently selected phenotypes. We observe similar patterns in our simulations: Increasing levels of mutational correlations and migration reduce differentiation between demes at the divergent phenotype, and increase differentiation between demes at non-divergent phenotypes. Perhaps surprisingly, this reduced phenotypic differentiation does not necessarily limit genetic repeatability, as high $C_{c h i s q}$ values are observed in simulations where pleiotropy and migration have substantially limited the divergence between demes (fig. 2).

Our simulations make a number of assumptions that are almost certainly violated in natural populations exhibiting evolutionary convergence. Firstly, we assume complete orthology between QTL in replicates and that orthologous QTL retain corresponding effect magnitude and pleiotropic properties. In nature, divergence between species limits studies of convergence to the orthologous portions of their genomes and the effects of adaptation in non-orthologous regions has not been addressed here. Secondly, we have assumed that both the initial phenotypic optima (to which both demes start our simulations adapted) and the divergent phenotypic optima are identical. Related species adapting to similar environments will not share identical phenotypic optima, which is important for the interpretation of our results, as Thompson, Osmond \& Schluter (2019) observed that repeatability declines rapidly as the angle between phenotypic optima increases, a pattern that is exacerbated by increased trait dimensionality. Furthermore variation between QTL in mutation rate, retention of standing variation and patterns of linkage disequilibrium may all affect the likelihood of repeatability, but we have held these parameters constant in our simulations.

The simulations presented here also use a simplified genome architecture: four QTL with

291 uniform properties and a single QTL with aberrant properties, and between two and ten

292 pleiotropic traits. This system pales in comparison to the thousands of genes (exhibiting near-

293 global pleiotropy) which contribute to traits under the omnigenic model (Boyle, Li \& Pritchard 
294 2017; Liu, Li \& Pritchard 2019). Contrastingly, a metaanalysis of gene knockout experiments in 295 Saccharomyces cerevisiae, Caenorhabditis elegans and Mus musculus (Wang, Liao \& Zhang 296 2010) estimated pleiotropy to be far less pervasive: a median gene affects only one to nine 297 percent of traits. Wang, Liao \& Zhang (2010) also detected significant signals of modular 298 pleiotropy (where subsets of genes affect subsets of traits), which would serve to simplify the 299 architecture available for evolutionary convergence. Simple genetic architecture enhances 300 repeatability at a genome-wide level, and this study demonstrates that an even more modular 301 architecture at some QTL will act to further magnify repeatability. While the nature of 302 pleiotropic, quantitative traits in higher organisms remains unresolved, we expect our simple 303 model to be applicable to more complex architectures (Yeaman et al. 2018), and repeating our 304 simulations on models with 20 QTL yields comparable results (fig. S3). 


\section{References}

306 Boyle, E.A., Li, Y.I. and Pritchard, J.K., 2017. An expanded view of complex traits: from 307 polygenic to omnigenic. Cell, 169(7), pp.1177-1186.

Chevin, L.M., Martin, G. and Lenormand, T., 2010. Fisher's model and the genomics of adaptation: restricted pleiotropy, heterogenous mutation, and parallel evolution. Evolution: International Journal of Organic Evolution, 64(11), pp.3213-3231.

Conte, G.L., Arnegard, M.E., Peichel, C.L. and Schluter, D., 2012. The probability of genetic parallelism and convergence in natural populations. Proceedings of the Royal Society $B$ : Biological Sciences, 279(1749), pp.5039-5047.

Doebley, J., 2004. The genetics of maize evolution. Annu. Rev. Genet., 38, pp.37-59.

Fisher, R.A., 1918. The correlation between relatives on the supposition of mendelian inheritance. Transactions of the Royal Society of Edinburgh, 52, pp 399-433.

Fisher, R.A., 1930. The Genetical Theory of Natural Selection. The Clarendon Press.

Haller, B.C. and Messer, P.W., 2019. SLiM 3: forward genetic simulations beyond the WrightFisher model. Molecular biology and evolution, 36(3), pp.632-637. 
McKenzie, J.A. and Batterham, P., 1994. The genetic, molecular and phenotypic consequences of selection for insecticide resistance. Trends in Ecology \& Evolution, 9(5), pp.166-169. adaptive evolution. Evolution, 52(4), pp.935-949.

Orr, H.A., 2005. The probability of parallel evolution. Evolution, 59(1), pp.216-220.

Rosenblum, E.B., Hoekstra, H.E. and Nachman, M.W., 2004. Adaptive reptile color variation and the evolution of the MCIR gene. Evolution, 58(8), pp.1794-1808.

Schlenke, T.A. and Begun, D.J., 2004. Strong selective sweep associated with a transposon insertion in Drosophila simulans. Proceedings of the National Academy of Sciences, 101(6), pp.1626-1631.

Schluter, D., 1996. Adaptive radiation along genetic lines of least resistance. Evolution, 50(5), pp.1766-1774.

Shapiro, M.D., Marks, M.E., Peichel, C.L., Blackman, B.K., Nereng, K.S., Jónsson, B., Schluter, D. and Kingsley, D.M., 2004. Genetic and developmental basis of evolutionary pelvic reduction in threespine sticklebacks. Nature, 428(6984), pp.717-723. Research, 23(1), pp.23-35.

Visscher, P.M., Wray, N.R., Zhang, Q., Sklar, P., McCarthy, M.I., Brown, M.A. and Yang, J., 2017. 10 years of GWAS discovery: biology, function, and translation. The American Journal of Human Genetics, 101(1), pp.5-22.

Wang, Z., Liao, B. Y., \& Zhang, J. (2010). Genomic patterns of pleiotropy and the evolution of complexity. Proceedings of the National Academy of Sciences, 107(42), 18034-18039.

Yeaman, S. and Whitlock, M.C., 2011. The genetic architecture of adaptation under migrationselection balance. Evolution: International Journal of Organic Evolution, 65(7), pp.1897-1911. 
bioRxiv preprint doi: https://doi.org/10.1101/2021.09.13.459985; this version posted September 13, 2021. The copyright holder for this preprint (which was not certified by peer review) is the author/funder, who has granted bioRxiv a license to display the preprint in perpetuity. It is made available under aCC-BY-NC-ND 4.0 International license.

372

373 Yeaman, S., Gerstein, A.C., Hodgins, K.A. and Whitlock, M.C., 2018. Quantifying how

374 constraints limit the diversity of viable routes to adaptation. PLoS genetics, 14(10), p.e1007717. 


\section{Supplementary figures}
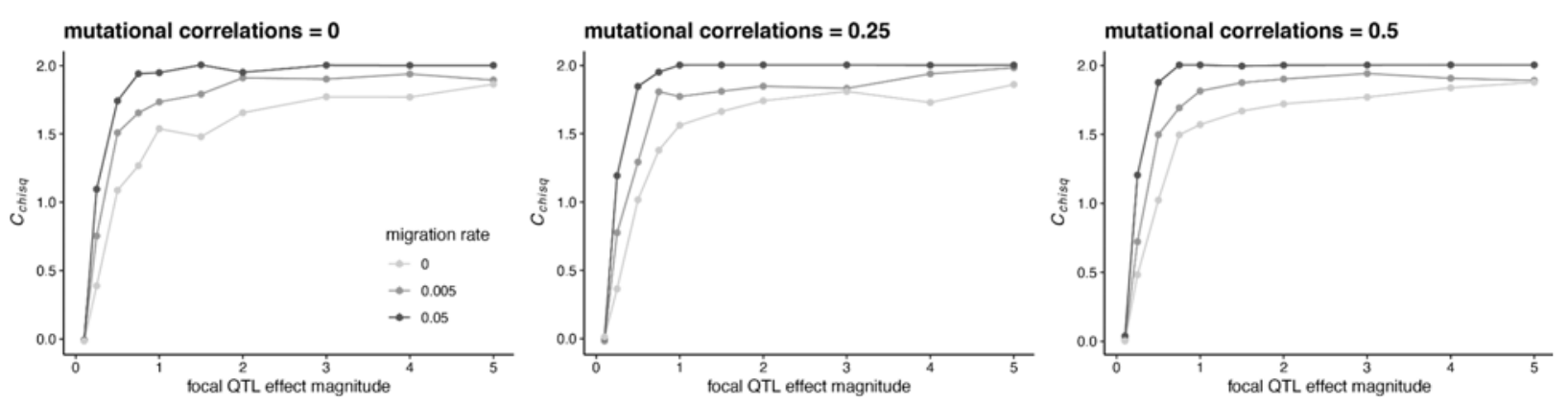

mutational correlations $=0.75$

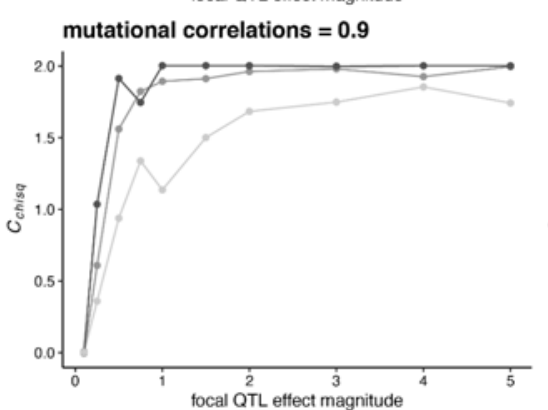

mutational correlations $=0.99$
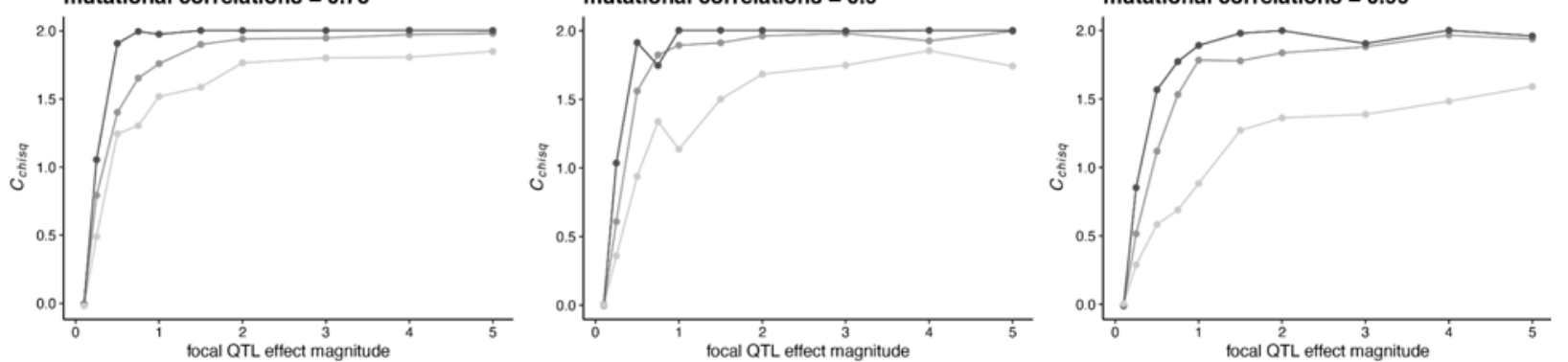

377 Figure S1. Repeatability $\left(C_{c h i s q}\right)$ in $Z_{i}$ against focal QTL effect magnitude where the effect magnitude for

378 non-focal QTL is 0.1 . These simulations use two phenotypes (one divergent and one non-divergent), and

379 were run for 20,000 generations. 

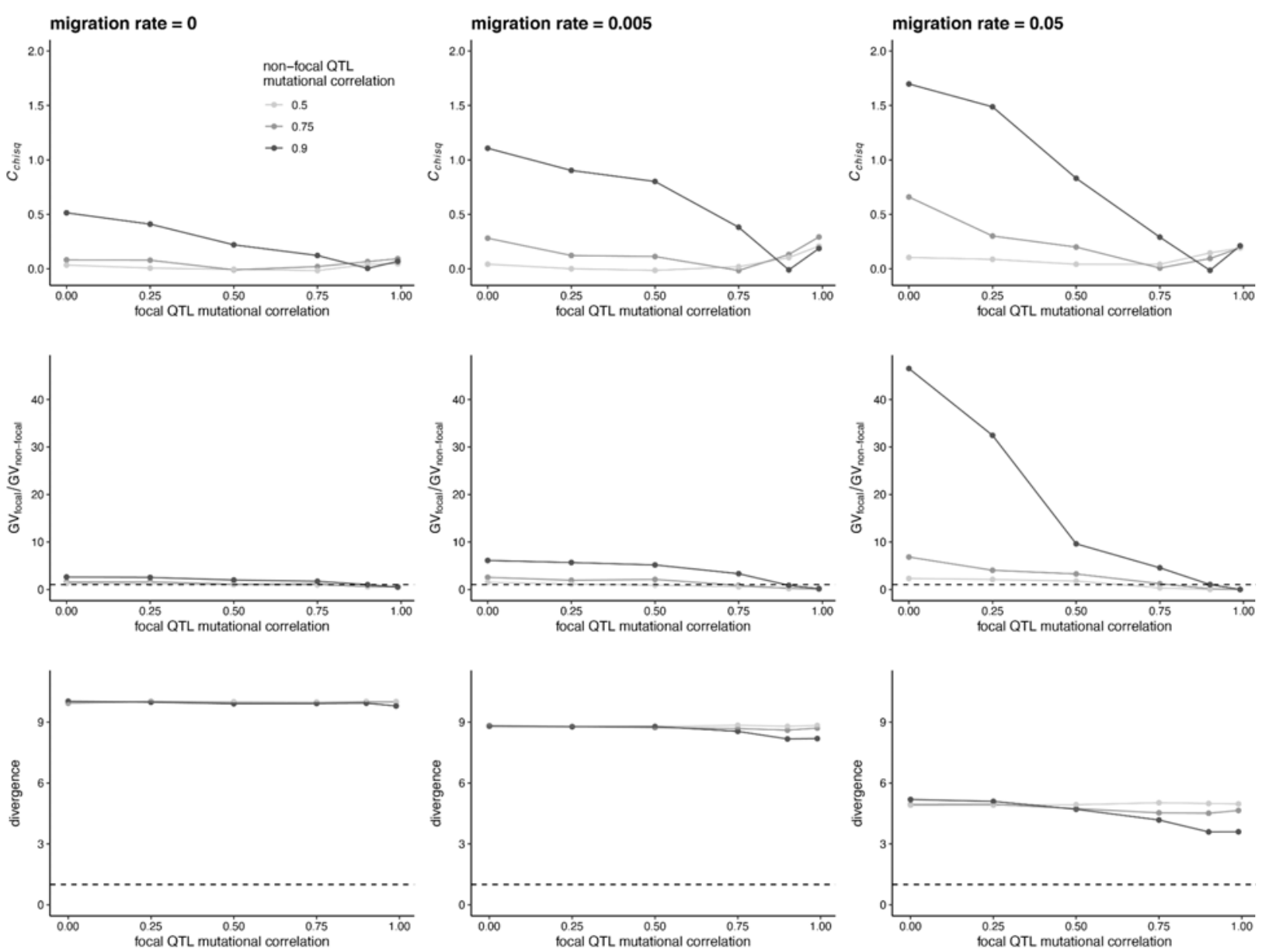

Figure S2. Repeatability $\left(C_{c h i s q}\right)$ in $Z_{i}$ against focal QTL mutational correlation for varying values of nonfocal QTL mutation correlation (top row), corresponding ratios of the $G V$ at focal QTL to mean $G V$ at nonfocal QTL (middle row), and divergence between demes (bottom row). These simulations use a mutation effect magnitude of 0.5 and two phenotypes (one divergent and one non-divergent), and were run for

38520,000 generations. 


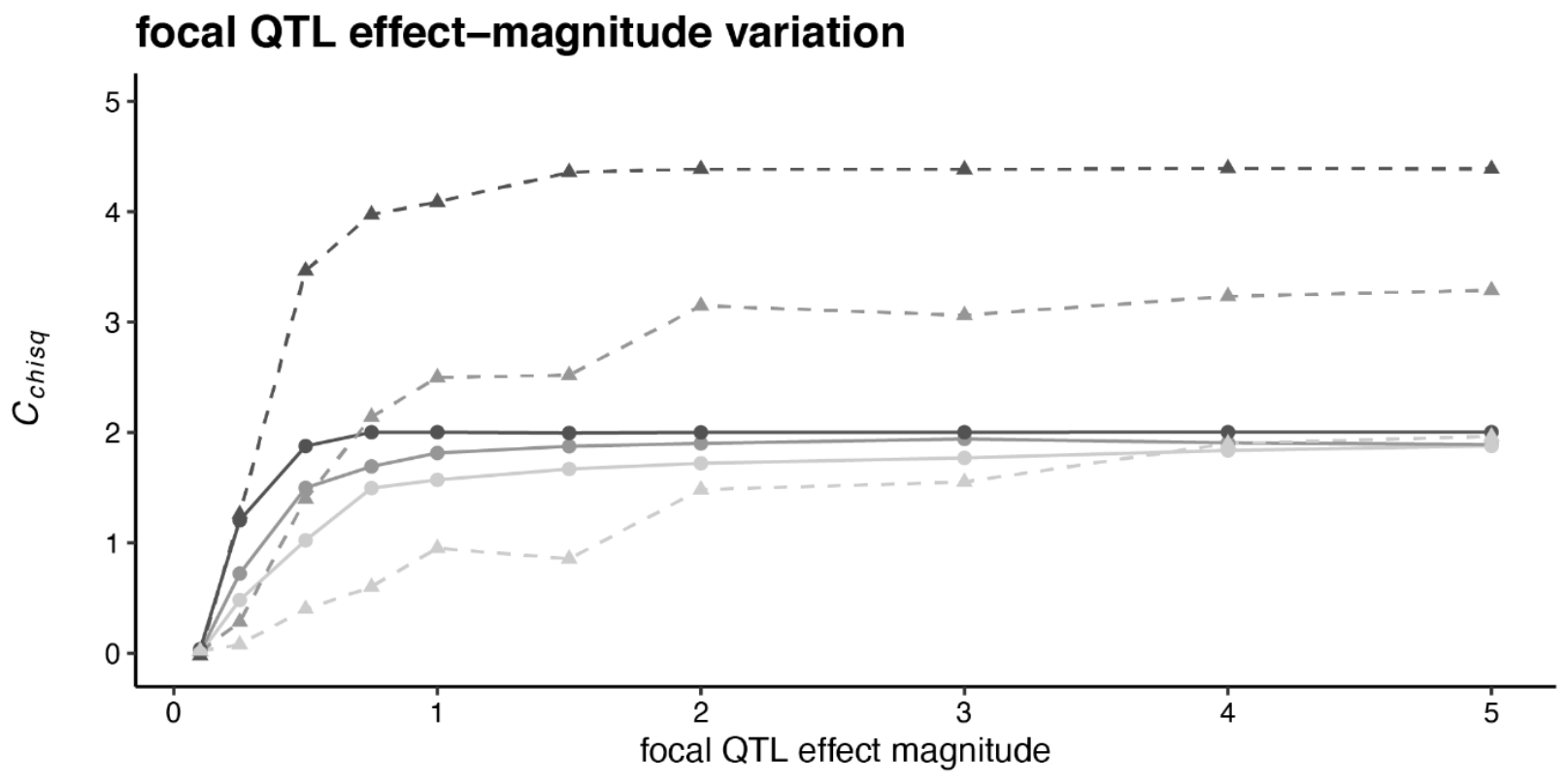

focal QTL mutational correlation variation

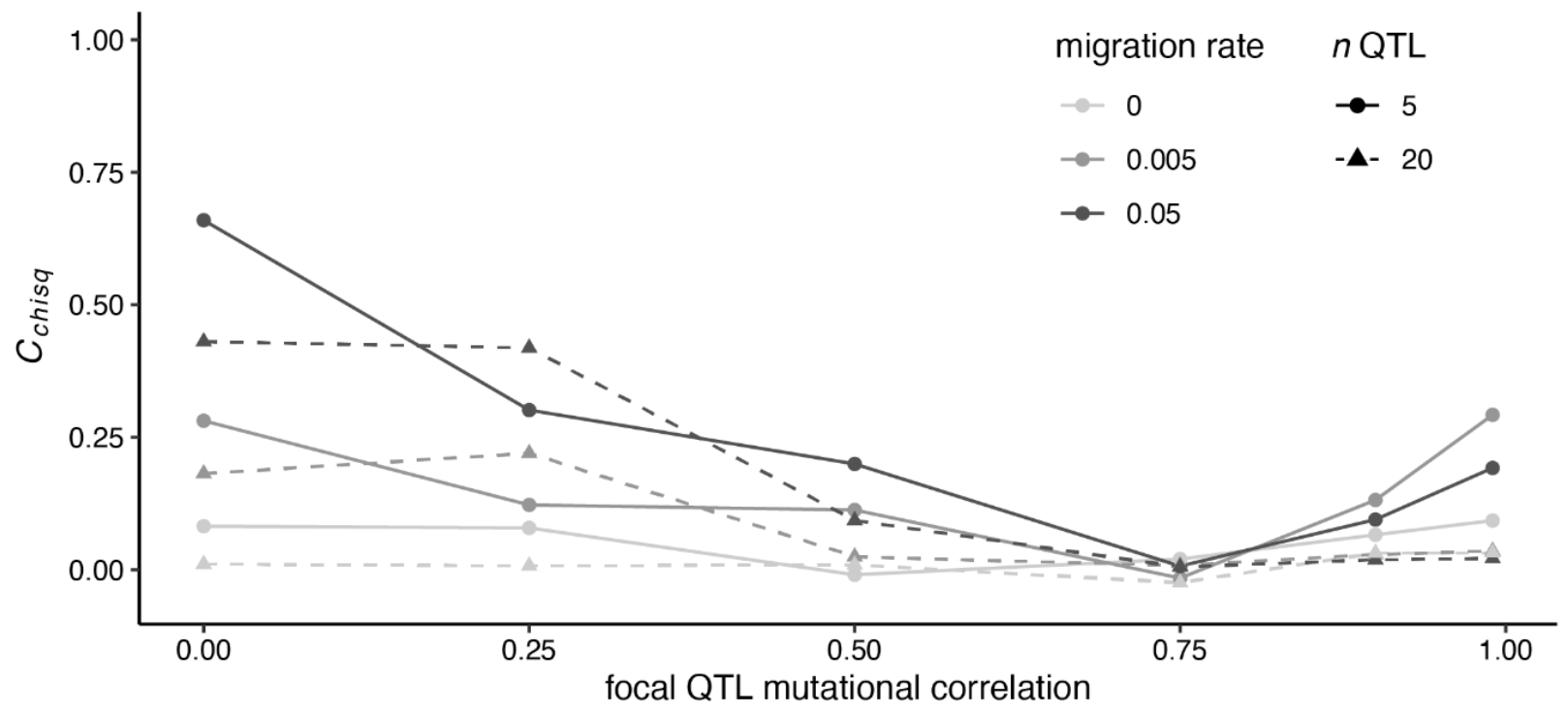

focal QTL dimensionality reduction

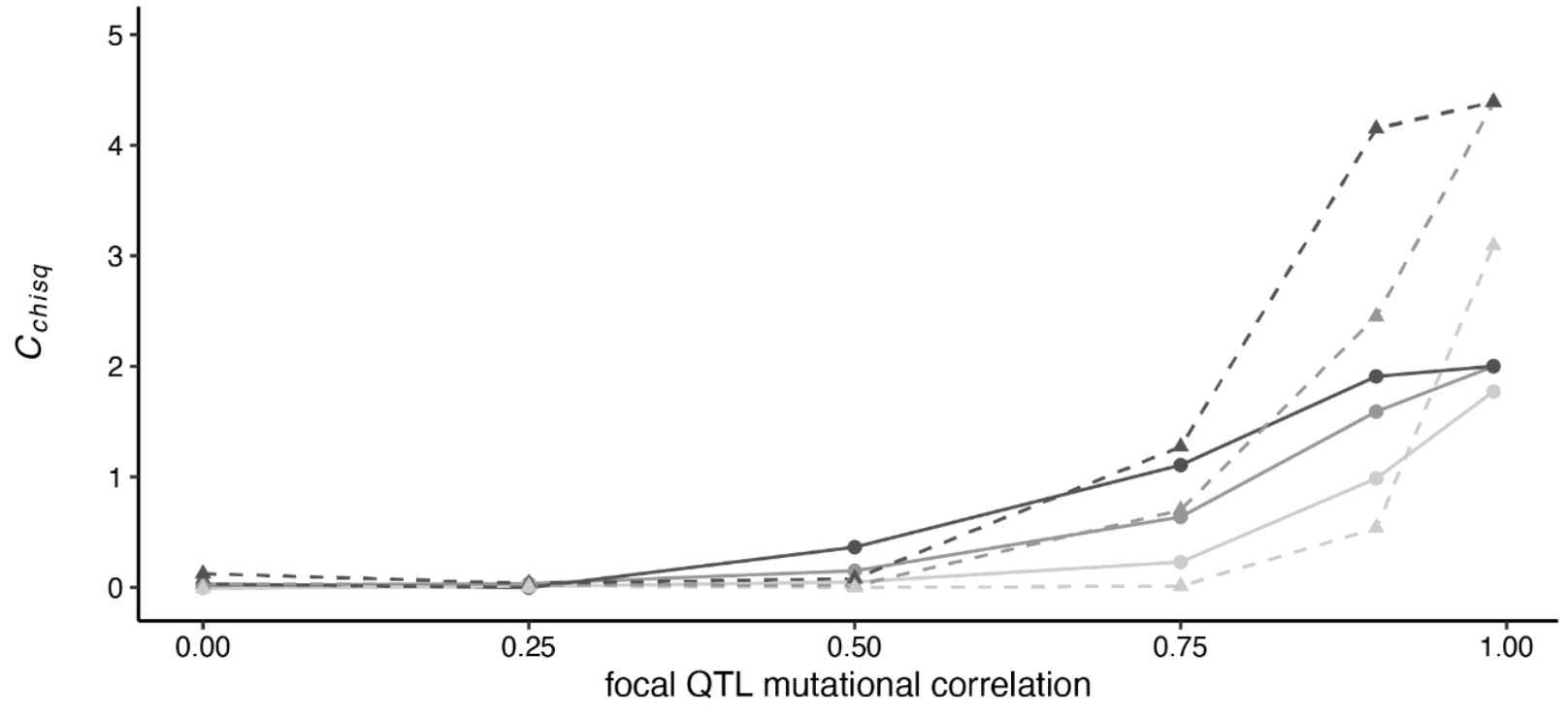


387 Figure S3. Effects of increasing the number of QTL modelled from five (solid lines, circle points) to 20

388 (dashed lines, triangle points). In the top pane we examine effect-magnitude variation at the focal QTL

389 (as in fig. 2), with mutational correlations for all QTL fixed at 0.5. In the middle pane we examine

390 mutational correlation variation at the focal QTL (as in fig. 3), with mutational correlations at non-focal

391 QTL of 0.75 and QTL effect magnitudes at 0.5 . In the lower pane we examine a reduction in

392 dimensionality at the focal locus (as in fig. 4B), where the total number of phenotypes is two and QTL

393 effect magnitudes are 0.5 . 